\title{
Subjective Unpossessed Reasons
}

\author{
Artūrs Logins \\ University of Fribourg
}

A common assumption in contemporary debates about normative reasons is that 'subjective' and 'possessed' are two names for the same sort of reason. This paper challenges that assumption. Given our cognitive limitations, it is unsurprising that normative reasons that derive from what we know and reasons that we are in a position to use in our deliberation are not always one and the same.

Keywords reasons; normative reasons; possession of reasons; contextualism about normative reasons; epistemology of reasons; deliberation

DOI:10.1002/tht 3.395

\section{Introduction}

Contemporary philosophy of reasons is characterized by an increasing number of notions and distinctions. While it is certainly true that some crucial notions are universally accepted (such as the distinction between normative and motivating reasons), it is also true that recent reasonology debates have become increasingly idiosyncratic. One response to such idiosyncrasy might be to appeal to Ockham's razor by investigating whether and how some notions within the debate can be reduced to others. So, for instance, instead of having both the concept of motivating reasons and the concept of operative reasons, philosophers are happy to accept that, in fact, people have used two different names for the same concept.

In such a context, the tendency is to assume the same kind of reduction with respect to subjective reasons (as opposed to objective) and possessed reasons (as opposed to unpossessed). According to this assumption, there is no distinction between possessed reasons and subjective reasons at all: thus, there are no reasons that are subjective but not possessed, and equally no reasons that are possessed but not subjective. Rather, just as 'motivating' and 'operative' are two names for the same thing, that is, a certain sort of reason, so are 'subjective' and 'possessed' two names for a sort of normative reason. ${ }^{1}$ Here are two (explicit) examples attesting to the popularity of the identification of subjective with possessed reasons:

... But in some sense or other, Freddie [i.e. the guy, who likes to dance and knows that there is going to be dancing at the party], unlike Ronnie [i.e. the guy who likes to dance but has no clue about the party], has this reason [to go to the party], since he 
knows about it, and Ronnie does not. This second sense of "has a reason" is the one I will later distinguish as the subjective sense of "reason". (Schroeder 2008, p. 59).

I reject the Factoring Account, so I deny that subjective reasons are a subset of objective reasons. I also deny that the status of something as a subjective reason is independent of its being possessed (Smithies 2018, p. 20, fn 29). ${ }^{2}$

Not everyone within the debate accepts that there are any objective/unpossessed normative reasons. Some think that all normative reasons are subjective/possessed. Yet, even in this case, the assumption is often that the distinction makes sense, and in particular, that 'subjective' and 'possessed' are merely two different names for one set of normative reasons whereas 'unpossessed/objective' (or perhaps simply 'unpossessed'3) refers to another (possible) set of normative reasons. That is, it makes sense to accept a distinction among two sorts of reasons even if, in fact, nothing falls under one term or the other.

The aim of this paper is to challenge the identification of subjective with possessed. I will argue that subjective reasons are distinct from possessed reasons. More precisely, I will argue that while there are possessed subjective reasons, there are also unpossessed subjective reasons. ${ }^{4}$ The distinction between subjective and possessed reasons is not like the (merely apparent) distinction between motivating and operative reasons. In what follows, I will first review the basics of one theoretical framework that appears to imply the distinction between subjective and possessed reasons. Then, I propose intuitive considerations in favor of the distinction, before indicating some of its further theoretical implications.

\section{A background theory}

To keep things clear, let us focus on the following senses of 'possessed' and 'subjective' reasons:

Possessed reasons (PR): a reason $r$ for $S$ to $F$ is possessed by $S$, just in case, $S$ is in a position to use $r$ in deliberation. ${ }^{5}$

Subjective reasons (SR): a reason $r$ for $\mathrm{S}$ to $\mathrm{F}$ is a subjective normative reason for $\mathrm{S}$ to $\mathrm{F}$ just in case $r^{\text {'s }}$ being a reason for $\mathrm{S}$ to $\mathrm{F}$ depends on $\mathrm{S}$ 's evidence. ${ }^{6}$

Three clarifications are in order here. First, note that ordinary English possessive constructions such as 'S has a reason to F' or 'S's reason to F' are extremely context sensitive and do not always capture the sense of 'possession' that is of interest for normative debates. ${ }^{7}$ Consider: (a) 'The building is on fire. She has a reason to leave.' The reason attribution here may be appropriate in a sense and, importantly, the two phrases may be true, even if the subject has no clue whatsoever about the ongoing fire. ${ }^{8}$ Crucially, the sense of 'having' reasons in (a) is not the one that we intend to capture by (PR) (nor by $[\mathrm{SR}]$ ). In short, the context sensitivity of possessive constructions calls for extra caution when relying on linguistic data to theorize about the possession of reasons. Our specification of 'possessed reasons' does not pretend to correspond to all possible uses of possessive constructions involving 'reasons'. 
Second, 'being in position to do something' is a context-sensitive expression. Consider, for instance, (i): "Carl is in position to prove Gödel's Incompleteness Theorem." The utterance (i) may well be true in a context where we focus on, say, comparing typical humans to some cognitively inferior animals. There is a sense in which a human, named Carl, and not, for instance, a jellyfish, is in position to prove the Incompleteness Theorem. This is a very weak sense, a sense that attributes the ability to prove the theorem to Carl merely because he is a human with a sophisticated cognitive capacity (and not a jellyfish). In a different context (i) will not come out as true. For instance, it will be false, in a context where we focus on Carl's ignorance in mathematics and logic. The sense of 'is in position to' that is relevant for our discussion here is not a weak one. We can follow Whiting, Hawthorne and Magidor (see footnote 5) and think of 'is in position to use $r$ in deliberation' as introducing an epistemic constraint. It requires that the subject's epistemic situation does not prevent the subject from using the relevant consideration in deliberation. Crucially, the mere fact that $\mathrm{p}$ follows from subject's evidence does not guarantee that the subject is in position to use $\mathrm{p}$ in her deliberation in the relevant sense. After all, one may believe or know that $\mathrm{p}$ and fail to know or even believe a proposition that follows from $\mathrm{p} .{ }^{9}$

Third, while 'depends' in (SR) is a bit vague, hopefully it is still clear enough for our purposes here. The crucial point is that 'depends' differs from 'consists of. More specifically, the point of (SR) is that we do not characterize one's subjective reasons as consisting only of one's evidence. Certain facts that are determined by one's evidence will count as subjective reasons on this specification even though they are not themselves part of one's evidence. One might think of a subject's evidence as a set of propositions that the subject knows (e.g., Williamson's $\mathrm{E}=\mathrm{K}$ thesis). ${ }^{10}$ Yet, what follows is also compatible with different views about evidence.

One theoretical framework that vindicates the distinction between subjective and possessed reasons is the view that takes seriously the context sensitivity of 'ought' and other modals. ${ }^{11}$ According to this linguistically informed approach, 'ought' can have different senses, since it is taken to order actions (and attitudes) according to a standard. ${ }^{12}$ There are objective oughts and subjective oughts. The former ranks actions (and attitudes) relative to what is best given all facts. Whereas the latter provides a ranking relative to what is expected to be best in the light of some agent's evidence (cf. Hawthorne and Magidor 2018 , p. 2). The suggestion then is to apply a similar line of thought to normative reasons, since the construction 'a reason/reasons to $\mathrm{F}$ ' is taken to encode the ought modality (cf. ibid) A natural conclusion is that 'a reason/reasons to $F$ ' is context sensitive in the same way as 'ought' is. This supports the idea that there are at least two sorts of normative reasons - objective and subjective. Subjective normative reasons are restricted by the subject's evidence, whereas, objective normative reasons are not restricted in this way. This approach does not identify subjective normative reasons with possessed normative reasons. For a consideration, $r$, to be a subjective normative reason for $\mathrm{S}$ to $\mathrm{F}$, just is for $r$ to speak in favor of F-ing for S, given the set of S's evidence. In numerous cases, when $r$ is a subjective normative reason for $\mathrm{S}, \mathrm{S}$ will possess $r$. Yet, the familiarity with such common cases need not lead us to the identification of the two. There might be cases 
where a consideration $r$ is a normative subjective reason for $\mathrm{S}$ to $\mathrm{F}$ (it is determined by $\mathrm{S}$ 's evidence), yet $S$ does not possess $r$, since $S$ is not in a position to use $r$ in her deliberation.

This was a quick summary of a theoretical view that supports the distinction between subjective and possessed reasons. However, we have not yet seen a convincing case that cannot be well understood unless we adopt the view in question. That is, now that we have filled in a conceptual space, we need some motivation for taking this option seriously. Does it actually have a useful application? The next section aims to explore this question.

\section{A case}

Consider an ordinary participant on the famous Let us Make a Deal show (also known as Monty Hall show). ${ }^{13}$ Let us call him Ben. He stands in front of three doors. There is a luxury car behind one of these doors and goats behind the two others. Ben has been given a chance to choose one of the three doors. Let us say Ben chooses door number 1 . Now, the show's host, Monty Hall, is obliged to open one of the three doors (that is the rule). Yet, he is not permitted to open the door that Ben has chosen. Neither can he open the door with the car behind it. Ben knows the rules of the game. Let us say Monty Hall opens door number 2. Of course, there is a goat behind it. Next, Ben is offered the chance to change his initial choice. That is, Ben can change his choice from door 1 to door 3 . Given what Ben knows, the thing to do (as long as he wants to win the car) is to switch to door 3. Given Ben's evidence, it is clearly more probable that the car is behind door 3. In fact, by opening door 2, Monty Hall gave Ben a crucial piece of information. Given the $1 / 3$ probability that Ben's first choice was the lucky one, there was a probability of $2 / 3$ that Monty Hall did not have any choice other than door 2 . That leaves a $2 / 3$ chance that the car is behind door 3. Now, it makes sense to think that the consideration $(r)$ 'it is more likely that the car is behind door 3 than door 1' (alternatively, 'the car is more unlikely to be behind door 1') is a normative reason for Ben to switch to door 3. That is, $r$ is a reason for Ben, in a sense. It may still be the case that the car is behind door 1. Hence, in another sense (an objective sense that is not tied to Ben's evidence) $r$ may also be no reason at all to switch. However, as long as we focus on the evidence that Ben possesses, $r$ is, intuitively, a normative reason (in a sense) for Ben to switch.

Crucially, Ben has 'the Monty Hall condition', that is, the condition of being unable to see that it is more probable that the car is behind door 3 than door 1 . As a matter of fact, a number of participants on the actual show were unable to see that, given what they knew, it was much more probable that the car was behind door 3 (that is, the door that was not initially chosen by the participant and was offered as a possible choice for a switch). It may take some time, repeated calculation, reading explanations and watching tutorials to overcome the 'Monty Hall condition' and finally be able to understand that it is more probable that the car is behind door 3 than door 1 . After all, it is common to describe the theoretical choice participants face on the show (when they have been offered the chance to switch a choice) as a problem or puzzle or even a paradox.

Assuming that Ben has the Monty Hall condition, we do not want to say that he possesses the consideration 'it is more likely that the car is behind door 3 than door 1' 
as a normative reason. For Ben is not in a position to use it in any kind of deliberation. Crucially, the 'being in position to' that is relevant here is an epistemic one, exactly as in (PR). Namely, there is something in Ben's epistemic position that prevents him from using the relevant consideration in his deliberation. After all, Ben does not believe and does not know that it is more likely that the car is behind door 3 than door 1 . The distinction between subjective normative reasons and possessed normative reasons seems to be the best way of making sense of the intuitive judgments about this case. The consideration 'it is more likely that the car is behind door 3 than door 1' is a subjective reason for Ben to switch, yet he does not possess it. It is an unpossessed subjective normative reason.

Those who collapse the distinction between the subjective and possessed normative reasons might object to the claim that $r$ is a normative reason for Ben to switch. One line of objection to this claim relies on the idea that only facts that determine (evidential) probability and not the probability facts (such as the fact that it is probable that $p$ ) can be reasons. According to this line of objection, only the relevant pieces of Ben's evidence are reasons for him to switch (for example that Monty opens door 2, that Monty cannot open door 1, and so on). The fact that, on Ben's evidence, it is more likely that the car is behind door 3 than door 1 is not a reason, according to this line of thought. ${ }^{14}$

However, this line of objection leads to unacceptable conclusions, since it would generalize to a myriad of ordinary considerations that we typically take to be our reasons to act. Giving up the idea that probability facts, such as that $p$ is probable, or that $p$ is more likely than $q$, can be reasons for a subject to $F$, would result in a massive denial of our ordinary intuitions. For example, consider a situation where it is likely, on my evidence, that it will rain in 5 minutes. Do we really want to say that we are not authorized to hold that the consideration 'it is likely on my evidence that it will rain' is a reason for me to close the windows or to look for my umbrella? To the contrary, the fact that the rain is very probable on my evidence speaks in favor of closing the windows or taking the umbrella. The probability fact here is a normative reason for me to act in certain ways.

The following examples may help illustrate this point further. Let us say that a mountain expedition has been organized, and participants are set up at the base camp and planing their ascent to the summit. Given what they know, it is $80 \%$ probable that there will be a heavy storm tomorrow. Do we want to say that this probability fact does not speak in favor of not planning the ascent for tomorrow? Given what his doctors know, it is more likely that a patient has the rare, severe and extremely contagious disease $\mathrm{X}$ than a simple flu. That probability fact speaks in favor of the doctors recommending the patient's immediate hospitalization. It is unlikely, given the available scientifically informed evidence, that a plague epidemic will break out in the city you live in anytime soon. It would seem odd to say that this consideration is not a reason for you to dismiss allegations of a major plague risk in your city. Rejecting the idea that probability facts can be normative reasons leads to an unwarranted skepticism about a large number of ordinary normative reasons.

Hence, I conclude that the Monty Hall example provides at least a prima facie support for the possibility of subjective unpossessed reasons. 


\section{Theoretical implications of the distinction}

Taking subjective unpossessed reasons seriously has further theoretical relevance. Here are two places where they might play a significant role.

A major theme in epistemology during the last 20 years or so is our presumed cognitive homelessness. Roughly, this view states that our inner life is not always available to us. According to an influential argument by Williamson (2000, pp. 93-113), there is no nontrivial condition $\mathrm{C}$, such that when $\mathrm{C}$ obtains, one can always know that one is in C. In particular, Williamson has argued that it is not the case that when we know that $p$, we always know that we know that $p$. His antiluminosity argument for that conclusion has received a sustained interest throughout the beginning of the 21st century. While it is far from being universally accepted, the antiluminosity argument has, nonetheless, moved contemporary mainstream epistemology towards taking the possibility of our cognitive homelessness more seriously.

The situation within the contemporary metanormative debates seems to be quite different in this respect. The fact that it is common not to distinguish between subjective and possessed normative reasons seems to reveal the prevailing tendency within the metanormative field to assume that people's inner lives, and in particular their inner normative lives, are always available to them. However, if what precedes is on the right track, then this common assumption needs to be revisited. The Monty Hall case shows that items within our inner (normative) life (such as subjective reasons) are not always available to us: even people's subjective reasons to act are not something that they are always in a position to know they have.

On a somewhat related note, much debate in contemporary epistemology has centered on the so-called internalism/externalism divide about epistemic justification. Even though the debate does not seem to be over yet, there are substantial lessons that epistemologists have already learned from it. One of these seems particularly relevant for our discussion, namely, that there are two ways of making the general characterization of internalism more precise. Internalist accessibilism is, roughly, the thesis that justification is determined by one's internal states that are accessible (to oneself) upon reflection alone. Whereas, internalist mentalism is the view that justification is determined by one's (internal) mental states, regardless of whether they are accessible or not. Now, it makes an important argumentative difference whether one defends internalist accessibilism or internalist mentalism, and epistemologists have noticed it. A similar contrast seems to apply to normative reasons as well. However, its importance has not yet been fully appreciated. More precisely, the above proposed distinction between possessed and subjective normative reasons parallels in a way the accessibilism/mentalism distinction about justification. One may think that practical rationality is determined by either one's subjective reasons, or by one's possessed reasons. These are two separate theses that the distinction between subjective-possessed reasons enables us to clearly separate. This matters, since one of these theses seems to be more demanding than the other. At least, certainly, the same arguments will not work for or against both of them. Overall, just as epistemologists have made progress by distinguishing accessibilism from mentalism, 
metanormative debates could benefit from taking the possessed/subjective reasons distinction more seriously. ${ }^{15}$

\section{Conclusion}

Philosophy of reasons has reached a point of increased terminological complexity. In such a situation, a natural tendency is to simplify the debate and reduce the number of postulated distinctions. One such tendency is to reduce subjective normative reasons to possessed normative reasons. I have argued here that such a tendency has to be resisted, or at least, not accepted from the outset. A respectable (yet, of course, debatable) and well understood theoretical framework licenses such a distinction. More importantly, some cases can be best understood with this distinction in place. Hence, simplifying the debate in this way has theoretical costs. A better strategy for advancing philosophy of reasons might be to pursue deeper theoretical issues, as many do, such as assessing the overall merits of contextualist approaches.

More fundamentally, what the Monty Hall case shows is that our cognitive limitations inevitably lead to the disparity between normative reasons that derive from what we know (or, at any rate, what we have as evidence) and normative reasons that we are in a position to use in deliberation. Not all of the former category also belong to the latter. Sadly, even when reasons are properly connected to a specific person, the person may not always be in a position to take advantage of that connection.

\section{Acknowledgments}

Many thanks to Davide Fassio, John Hawthorne, Travis Timmerman and anonymous referees for this journal for helpful comments and suggestions on earlier version of this paper. The research work that led to this article was supported by the Swiss National Science Foundation grant number 171464.

\section{Notes}

1 Hawthorne and Magidor (2018), is one rare place where this identification has been rejected. They rely on this rejection in their objection to Schroeder's arguments against the so-called Factoring Account of reasons. Sylvan (2016), also appears to distance himself from the identity view.

2 Here is an additional example: 'One common way of drawing the distinction [between 'objective' and 'subjective' reasons] is in terms of the reasons there are for some agent $\mathrm{S}$ to $\mathrm{A}$ (so-called "objective reasons") and the reasons S has to A (so-called "subjective reasons").' (Fogal and Sylvan 2017, p. 6, fn 6.) Note that Fogal and Sylvan do not endorse such a distinction themselves.

3 This is to indicate that I do not want to imply that the approach that I criticize in this paper has to assume that the sets of subjective and objective reasons are disjoint. My opponents may well hold that subjective reasons constitute a subset of objective reasons and that it makes sense to distinguish between possessed objective reasons (i.e., subjective reasons) and 
unpossessed objective reasons (i.e., merely objective reasons). Thanks to an anonymous referee for this suggestion.

4 While I believe that subjective/objective and possessed/unpossessed are orthogonal and not merely different distinctions, in what follows I will focus exclusively on the subjective (possessed/unpossessed) reasons.

5 Compare to: '[T] here is a familiar distinction between the reasons there are for a person to act and the reasons she possesses for acting, where a person possesses a reason for acting only if she is in a position to act for that reason.( ... ) A common suggestion is that to possess a reason requires standing in an epistemic relation to the relevant consideration' (Whiting 2018, p. 3). See also: 'The contrast between possessed and unpossessed reasons we have in mind is fairly intuitive. When a glass contains poison but an agent is unaware of this, there is a reason for the agent to avoid drinking from the glass, but that reason for avoidance is something that the agent is not in a position to use as a consideration when acting' (Hawthorne and Magidor 2018, p. 2).

6 See, for instance: 'Roughly our envisaged objective 'ought' ranks actions according to the best outcome, while the subjective 'ought' ranks according to the best expected outcome by the lights of the agent's evidence.(...) (We think of evidence as what the subject knows, though much of what we say could be adapted to other frameworks for thinking about evidence.) This basic structure covers both reasons to act and reasons to believe' (Hawthorne and Magidor 2018, p. 2). Compare to: 'Second, it is fairly common in the literature we are concerned with (and elsewhere) to distinguish between objective and subjective 'should's'. On a simple way of drawing this distinction, what you objectively should do is determined by the facts of your situation, whereas what you subjectively should do is determined by your perspective on your situation' (Way 2018, p. 14).

7 Thanks to an anonymous referee for reminding me of the importance of this context sensitivity.

8 See Hawthorne and Magidor (2018), for similar and more sophisticated examples. An anonymous referee proposes also the following example as an illustration of the context sensitivity in question: 'It turns out that we had good reason to proceed with caution, though of course we could not have known it at the time'. See also Fogal and Sylvan (2017), for further observations about the context sensitivity of the possessive constructions.

9 Thanks to an anonymous referee for drawing my attention to the need to clarify this issue.

10 Cf. Williamson (2000, pp. 184-237). See also, Logins (2017), among others for a recent defense of $\mathrm{E}=\mathrm{K}$.

11 The view sketched here has been proposed recently by Hawthorne and Magidor (2018).

12 A more precise formulation would appeal to the rankings of states of affairs. The linguistic theory in the background of this view comes from Kratzer (1981) (and elsewhere). This presentation is a very rough and incomplete one. A number of important elements need to be added here (as for instance, a reference to modal bases or domains of states of affairs that are ranked).

13 This case is well known (especially within the probability theory) as giving raise to the Monty Hall Problem, see vos Savant (1992, pp. 199-209).

14 Thanks to an anonymous referee for drawing my attention to this possible objection.

15 Note also that internalist accessibilism, as defended recently by Declan Smithies (cf. Smithies 2012), has to focus on possessed reasons to believe and not merely subjective ones (assuming that what justifies one to believe something are normative reasons to believe). Subjective 
reasons that are not possessed are not accessible and, hence, cannot determine justification according to internalist accessibilism. Therefore, it is unfortunate that Smithies fails to distinguish between possessed and subjective reasons (see the quote above). This makes his view problematically ambiguous between a reading that cannot be true, given the very formulation of accessibilism, and a reading that is internally coherent but very demanding. Thanks to an anonymous referee for drawing my attention to the problem that the subjective/possessed distinction applied to reasons to believe might raise for Smithies' accessibilist account.

\section{References}

Fogal, Daniel and Kurt Sylvan. "Contextualism about Epistemic Reasons," in The Routledge Handbook to Epistemic Contextualism, edited by Jonathan Jenkins Ichikawa. London: Routledge, 2017.

Hawthorne, John and Ofra Magidor. "Reflections on the Ideology of Reasons," in The Oxford Handbook on Reasons and Normativity, edited by Daniel Star. Oxford: Oxford University Press, 2018, $113-139$.

Kratzer, Angelika. "The Notional Category of Modality," in Words, Worlds, and Contexts: New Approaches in Word Semantics (Research in Text Theory 6), edited by Hans-Jürgen Eikmeyer and Hannes Rieser. Berlin: de Gruyter, 1981, 38-74.

Logins, Artūrs. "Common Sense and Evidence: Some Neglected Arguments in Favour of E=K." Theoria 83.2 (2017): 120 - 137.

Schroeder, Mark. “Having Reasons.” Philosophical Studies 139.1 (2008): 57-71.

Smithies, Declan. "Reasons and Perception," in The Oxford Handbook of Reasons and Normativity, edited by Daniel Star. Oxford: Oxford University Press, 2018.

Smithies, D. "Moore's Paradox and the Accessibility of Justification." Philosophy and Phenomenological Research 85.2 (2012): 273-300.

Sylvan, Kurt. "Epistemic Reasons I: Normativity." Philosophy Compass 11.7 (2016): 364-376.

vos Savant, M. Ask Marylin. New York: St. Martin's, 1992.

Way, Jonathan. "Reasons and Rationality," in The Oxford Handbook of Reasons and Normativity, edited by Daniel Star. Oxford: Oxford University Press, 2018.

Whiting, Daniel. "Right in Some Respects: Reasons as Evidence." Philosophical Studies 175.9 (2018): 2191-2208.

Williamson, Timothy. Knowledge and Its Limits. Oxford: Oxford University Press, 2000. 